\title{
Is a multiple excitation of a single atom equivalent to a single excitation of an ensemble of atoms?
}

\author{
Ido Kanter, Aviad Frydman and Assaf Ater \\ Minerva center and the Department of Physics, Bar Ilan University, Ramat-Gan 52900, Israel
}

\begin{abstract}
Recent technological advances have enabled to isolate, control and measure the properties of a single atom, leading to the possibility to perform statistics on the behavior of single quantum systems. These experiments have enabled to check a question which was out of reach previously: Is the statistics of a repeatedly excitation of an atom $\mathrm{N}$ times equivalent to a single excitation of an ensemble of $\mathrm{N}$ atoms? We present a new method to analyze quantum measurements which leads to the postulation that the answer is most probably no. We discuss the merits of the analysis and its conclusion.
\end{abstract}

PACS numbers: 03.65.Ta, 42.50.Lc, 02.50.Ga

The development of laser cooling techniques have enabled to study the properties of single atoms. This research is motivated both by the quest for better understanding of basic quantum mechanics concepts as well as by potential applications in the fields of quantum computers, quantum clocks and random number generators. 1, 2, 3]. Though the accuracy of quantum mechanical experiments is increasing rapidly, the analysis of the data is still in its primary stage and a method for detecting correlations is far from being established.

An example for the above effort is the advanced study of quantum jumps in a single atom. Figure 1 shows the results of a typical quantum jump experiment performed on a ${ }^{189} \mathrm{Hg}^{+}$ion (data provided by W.M Itano). The atomic system contains two excited states (as seen in figure 2). State 1 has a strong coupling to the ground state while state 2 is weakly coupled and has a long lifetime. If the system is excited to state 1 it will emit a photon as the system decays to the ground state ("light" level) unless it is transferred to state 2 in which case the fluorescence will stop for a period of the lifetime of this state ("dark" level). The data is viewed as a set of switches between two levels. The higher one representing detection of emitted photons as the sample decays from state 1 to the ground state and the lower one (basically 0 ) represents the situation where the sample is at state 2 .

This type of experiments opens the opportunity to examine new questions related to quantum mechanics. It is well established that the decay of an ensemble of quantum particles is governed by a Poissonian process. The number of decay events as a function of time is proportional to $\exp (-t / \tau)$, where $\tau$ is the relevant life time. This behavior indicates that the process is random with no correlations among successive events. Such statistics were confirmed in many quantum experiments such as Alpha and Beta radiation and decay of excited atoms to the ground state [4]. The question we raise in this work is whether the excitation of a single atom many times
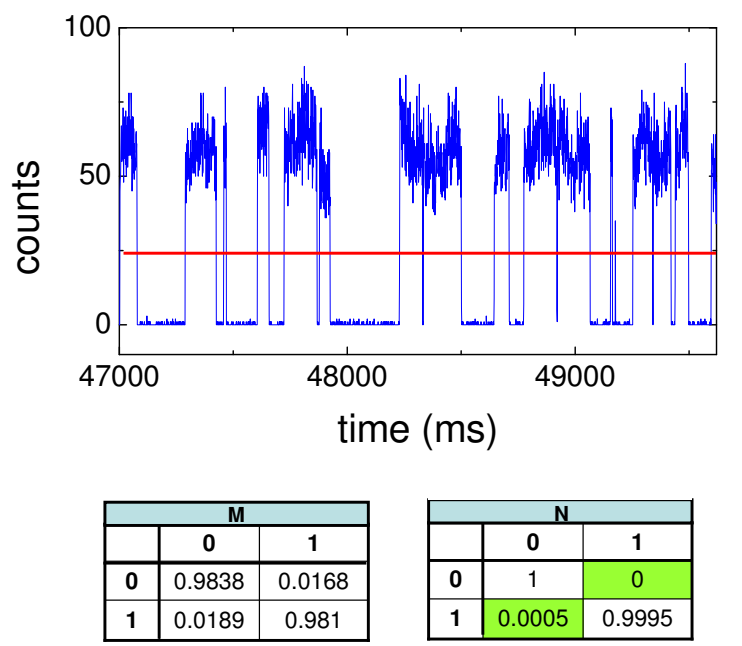

FIG. 1: A section of a typical quantum jump measurement performed on a Mercury ion. The red line shows the clipping threshold for the analysis process (results are insensitive to the precise threshold value). The figure also depicts the Markov matrix (M) and the noise matrix (N) obtained from the analysis of the entire experimental data set using by the BW algorithm.

would exhibit the same behavior and statistics. For instance, in the quantum jumps experiments, the question is whether the level lifetimes are random and temporally uncorrelated. This question is of major importance both for the foundation of quantum mechanics as well as for setting guidelines for future applications.

In order to check this question we develop a method to analyze the data of quantum jumps experiments. This method is based on the theory of the hidden Markov model [5, 6, 7]. A Markov model is a finite state machine that changes state once every time unit. The manner in which the state transitions occur is probabilistic and is governed by a state-transition matrix, M. For applying this model to quantum jumps we focus on the simplest case of a Markov model, i.e. only two levels "light" and 


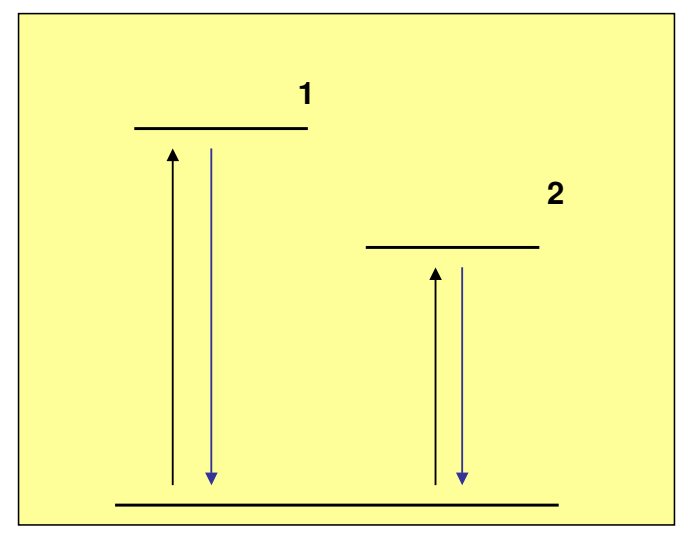

FIG. 2: A schematic energy diagram of the ionic system.

"dark". Quantum jumps are expected to be described by a Markovian process, since events are assumed to depend only on the current state and not on the history. If on the other hand, a system does not follow pure Markovian statistics, the process is named a Hidden Markov Process. For example, if the atom experiences external fields or interactions, one may expect to observe a deviation from Markovian behavior. In this case the process has to be described by two $2 X 2$ transition matrices. The first one, M, stands for the transitions due to the Markovian process $(\mathrm{M}(\mathrm{i}, \mathrm{j})$ stands for the transition from $\mathrm{i}$ to j) while the second one, N, represents unexpected transitions that can not be ascribed to the usual statistics of the system. The off-diagonal elements of matrix N, known also as the noise, measure the amount of deviation from pure Markovian behavior. For pure Markovian processes the off-diagonal elements are zero and as these elements increase the noise increases. A practical way to determine the two matrices, $\mathrm{M}$ and $\mathrm{N}$, that characterize the most likely underlying processes, is known as the Baum-Welch (BW) algorithm [5].

The actual analysis of the quantum jumps data is performed using the following procedure. A data set, such as that of figure 1 , is clipped to produce a symbolic data sequence containing two levels, 0 and 1 (0 representing a "dark" level and 1 representing the "light" level). The transitions between these levels are analyzed by running the Baum-Welch procedure [5] to produce two matrices, $\mathrm{M}$, and, N. A typical result is shown in figure 1. Here we analyze the data of a sequence of $10^{5}$ data points where each point represents the number of photon counts in a time interval of $1 \mathrm{~ms}$.

The off-diagonal elements of matrix $\mathrm{N}$ show that while there are no unexpected transitions from $0 \rightarrow 1$, there are non-trivial transitions from $1 \rightarrow 0$ that are not consistent with a simple Markovian framework. It is important to

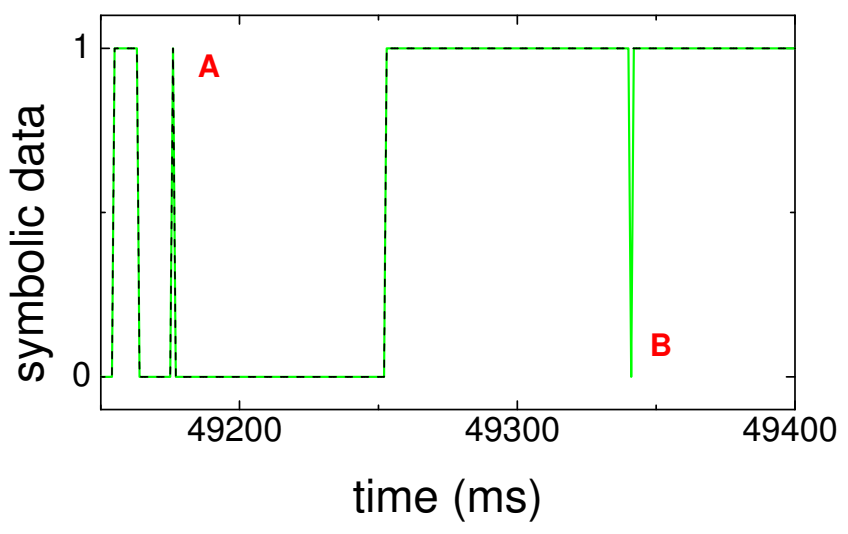

FIG. 3: Part of the symbolic data sequence after clipping the data of figure 1 . The dashed line shows the result of the Viterbi algorithm.

note that though the effect is rather small, it can not be attributed to a finite size artifact. We have generated artificial sequences (using matrix $\mathrm{M}$ only) having sizes similar to the experimental one which produced pure Markov matrices with negligible off-diagonal elements of matrix N. Similar non-Markovian results were obtained on a longer sequence $\left(7.5 * 10^{5}\right.$ data points $)$ taken from a Sr quantum jumps experiment [2].

The BW procedure indicates that a single ion produces unexpected transitions. One may ask whether it is possible to go one step further and to identify the location (in the time sequence) of the noise. Such a question arises, for instance, in the case of digital communication, where a Markovian message is transmitted through a noisy channel. The receiver's goal is to identify the locations of the noisy bits in order to recover the original signal [8]. An established way to do this is by using the Viterbi algorithm [9] to compute the most likely underlying Markov sequence, or, in other words, the expected physical outcome without the detected noise [10].

The outcome of running the Viterbi algorithm on the data of figure 1 is presented in figure 3 . The clipped data contains sub-sequences like ...000010000... (type A) or ...111101111.... (type B). The Viterbi procedure identifies most of events of type B as noise, but non of type A. A similar effect was obtained in all studied quantum jump experimental sequences (over 10 data sets).

The above result reveals the fact that sawtooth-like features of type B ( $\mathrm{a}$ few dozens in a sequence of $10^{5}$ data points), can not be explained as part of the usual statistics of the ion, i.e. Markovian statistics. We demonstrate 
that these sawteeth are the cause for non-Markovian noise by flipping these particular data points from 0 to 1 and running the BW procedure on the revised sequence. This procedure yields practically pure Markov matrices even for flipping only $\sim 40 \%$ of the features detected by the Viterbi process as problematic.

It is important to note that deviations from pure exponential behavior of the plateau statistics were observed in quantum jumps experiments [2]. These were attributed to experimental difficulties in controlling the laser amplitude and frequency over relatively large time periods. This experimental artifact gives rise to non-Poissonian statistics due to long plateaus (with times larger than $130 \mathrm{~ms}$ ). Our analysis (the BW and the Viterbi algorithms) implies that it is the short plateaus, rather then the long ones, which are responsible for the unexpected behavior. Further support is obtained by running the BW algorithm on quantum jump data once while erasing long plateaus (having time scales above 100ms) and again while erasing plateaus shorter than $2 \mathrm{~ms}$. In the former case we found that the off-diagonal noise remained non-zero. On the other hand the latter case revealed that the noise was entirely suppressed when the short plateau were removed from the sequence. Our analysis therefore strongly implies that the sources of non-Markovian behavior in a single atom experiment are the short plateaus where the system spends a relatively short time at the excited state.

In an attempt to understand the results of the analysis we note that though an atom is a single quantum particle it is a many body system containing many degrees of freedom. Therefore, the decay of the atom to the ground state may have some characteristic timescales. This might be a source for our observations. If the ion is repeatedly excited with very short time intervals it may not be able to fully relax. This may give rise to a deviation from the expected Markovian statistics. In this respect the excitation of a single atom many times may differ from a single excitation of many atoms. A natural way to further check this hypothesis experimentally is to enhance the effect by taking measurement with a shorter characteristic timescale (counting photons in time intervals shorter than a ms). We suspect that such measurements would result in a larger deviation from Markovian behavior.

We gratefully acknowledge W.M Itano and D. J Berkeland for providing the quantum jump experimental data and P.W. Anderson and L. Khaikovich for useful discussions.

[1] C.E. Wieman, D.E. Pritchard and D.J. Wineland, Rev. Mod. Phys., 71, S253 (1999).

[2] D.J. Berkeland, D.A. Raymonson and V.M. Tassin, arXiv : physics0304013.

[3] S.A. Diddmas et. al., Science 293, 825 (2001).

[4] For example see A.G. Calamai and C.E. Johnson, Phys. Rev. A 42, 5425 (1990).

[5] G.J. McLachen and T. Krishnan, "The EM Algorithm and Extensions", John Wiley and Sons (1997).

[6] L.B. Rabiner, B.H. Juang, "An Introduction to Hidden Markov Models", IEEE ASSP Magazine, vol 3, February 1986.

[7] I. Kanter, A. Frydman and A. Ater, Cond - mat/0402246.

[8] T.M. Cover and J.A. Cover, "Elements of Information Theory", John Wiley and Sons (1991).

[9] J.G. Proakis, "Digital communication", McGraw-Hill (2001).

[10] Note that the separation of the received message to signal and noise is not always possible due to either theoretical bounds of information theory or limitations of the used algorithm and the examined sequence. However, in many cases the Viterbi algorithm proves very usuful. 